固相萃取-高效液相色谱法测定地表水中的苯并三唑和苯并噻唑

$$
\begin{aligned}
& \text { 王金成 }{ }^{1} \text {, 熊 } 力^{2} \text {, 张海军 }{ }^{1} \text {, 王龙星 }{ }^{1} \text {, 金 静 }{ }^{1} \text {, 陈吉平 }{ }^{1} \text { ( } \\
& \text { (1. 中国科学院大连化学物理研究所, 辽宁 大连 } 116023 ; 2 \text {. 大连工业大学, 辽宁 大连 116034) }
\end{aligned}
$$

摘要: 建立了固相萃取-高效液相色谱分析地表水中苯并噻唑、苯并三唑、5-甲基-苯并三唑、5-氯-苯并三唑及 5,6-二 甲基-苯并三唑的方法。地表水样品采用 HLB 固相萃取柱富集净化后, 在甲醇-水 $(55: 45, \mathrm{v} / \mathrm{v})$ 流动相中用 ZORBAX SB-C18 色谱柱 $(250 \mathrm{~mm} \times 4.6 \mathrm{~mm}, 5 \mu \mathrm{m})$ 分离, 紫外检测, 外标法定量。结果表明,5 种目标化合物在 $0.064 \sim$ $80 \mathrm{mg} / \mathrm{L}$ 范围内均呈良好的线性关系, 线性相关系数均大于 0.9999 , 仪器检出限为 $1.9 \sim 3.2 \mu \mathrm{g} / \mathrm{L}$ 。空白自来水 样中的加标回收率为 $87.8 \% \sim 125.6 \%$, 相对标准偏差 $(n=3)$ 为 $0.4 \% \sim 9.4 \%$ 。应用该方法测定了大连自来水及辽 河人海口水样,在辽河人海口地表水中检出苯并噻唑、苯并三唑、5-甲基-苯并三唑、5-氯-苯并三唑。

关键词: 固相萃取;高效液相色谱法;苯并三坐; 衍生物;苯并噻唑;地表水

中图分类号: O658 文献标识码:A 文章编号:1000-8713(2013)02-0139-04

\title{
Simultaneous determination of benzotriazoles and benzothiazole in surface water by solid phase extraction and high performance liquid chromatography
}

WANG Jincheng ${ }^{1}$, XIONG Li $^{2}$, ZHANG Haijun ${ }^{1}$, WANG Longxing ${ }^{1}$, JIN Jing ${ }^{1}$, CHEN Jiping ${ }^{1}$

(1. Dalian Institute of Chemical Physics, Chinese Academy of Sciences, Dalian 116023, China;

2. Dalian Polytechnic University, Dalian 116034, China)

\begin{abstract}
A simple method based on solid phase extraction (SPE) coupled with high performance liquid chromatography ( HPLC) was developed for the determination of $1 \mathrm{H}$-benzotriazole (BT) , 5-methyl-1 $H$-benzotriazole (5-M-BT) , 5,6-dimethyl-1 $H$-benzotriazole (5,6-DM-BT) , 5chloro-1H-benzotriazole (5-Cl-BT) and benzothiazole (BTH) in surface water. The extraction and clean-up of the surface water sample were performed on an HLB solid-phase extraction cartridge. The separation of the five compounds was achieved on a ZORBAX SB-C18 column (250 $\mathrm{mm} \times 4.6 \mathrm{~mm}, 5 \mu \mathrm{m})$ with methanol-water $(55: 45, \mathrm{v} / \mathrm{v})$ as mobile phase and ultraviolet detection. The quantification was achieved by external standard method. The results showed that good linearities were observed in the ranges of $0.064-80 \mathrm{mg} / \mathrm{L}$ with the correlation coefficients $\geqslant 0.9999$ and the instrumental limits of detection for the five target compounds were in the range of $1.9-3.2 \mu \mathrm{g} / \mathrm{L}$. The average recoveries of the five target compounds were $87.8 \%-$ $125.6 \%$ and the relative standard deviations $(n=3)$ were $0.4 \%-9.4 \%$. This method was applied to the analysis of the surface water samples from the Dalian tap water and the Liaohe River estuary. The results showed that BT, 5-M-BT, 5-Cl-BT and BTH were found in the water from the Liaohe River estuary.
\end{abstract}

Key words : solid phase extraction (SPE) ; high performance liquid chromatography (HPLC) ; benzotriazoles; derivatives; benzothiazole; surface water

苯并三唑和苯并噻唑是工业生产和家庭生活中 广泛使用的高产量的化工产品。苯并三唑及其甲基
衍生物对金属具有防腐缓蚀作用,被广泛用于飞机 除冰剂、发动机防冻剂、机动车制动液、金属切割液 
及工业循环冷却水系统 ${ }^{[1-3]}$; 苯并噻唑常用于橡胶 工业生产过程中的促进剂 ${ }^{[4]}$, 另外也广泛用于防腐 剂 ${ }^{[5]}$ 及化工中间体 ${ }^{[6]}$ 等。

由于苯并三唑和苯并噻唑类化合物为水溶性, 且生物降解性及吸附能力均较差, 因此经过废水处 理厂处理后的工业和生活废水中只有少部分苯并三 唑和苯并噻唑类化合物被处理掉, 大多数被排放到 江河湖海中 ${ }^{[7-9]}$ 。目前有关这类化合物的毒性报道 还很少,但有研究表明苯并三唑类化合物对水生物 有一定的毒性, 而且有生物累积作用 ${ }^{[2]}$ 。也有证据 表明苯并三唑可能对人类有致癌作用 ${ }^{[10]}$ 。美国环 境保护署已连续多年将苯并三唑列为新兴污染 物 ${ }^{[11-13]}$ 。澳大利亚政府规定饮用水中苯并三唑的 质量浓度不能超过 $7 \mathrm{ng} / \mathrm{L}^{[14]}$ 。因此测定环境水中 苯并三唑和苯并噻唑类化合物的浓度, 对研究这一 类化合物的毒性及迁移转化规律有一定的意义。

目前测定苯并三唑和苯并噻唑类化合物的方法 有气相色谱-质谱法 ${ }^{[15,16]}$ 、液相色谱-质谱法 ${ }^{[6,8]}$ 及液 相色谱-串联质谱法 ${ }^{[7,17-20]}$ 等。国内有关环境中这 两类化合物的测定尚未见报道。本研究采用固相萃 取-高效液相色谱法测定地表水中苯并三唑和苯并 噻唑类化合物,并进行了方法学评价。

\section{1 实验部分}

\section{1 仪器与试剂}

Agilent 1200 型高效液相色谱仪 (美国安捷伦 公司)。苯并三唑(1H-benzotriazole，BT)、5-甲基 苯并三唑 (5-methyl-1H-benzotriazole，5-M-BT) 、 5,6-二甲基苯并三唑 ( 5,6-dimethyl-1H-benzotriazole, 5, 6-DM-BT) 购于 ACROS ORGANICS, 5氯-苯并三唑 (5-chloro-1 $H$-benzotriazole, 5-Cl-BT) 购于日本东京化成工业株式会社, 苯并噻唑 (benzothiazole, BTH) 购于百灵威化学试剂有限公 司,以上试剂纯度均大于 $98 \%$ 。甲醇 (色谱纯, 美国 Fisher 公司); 盐酸及氢氧化钠为分析纯; 实验用水 为经 Milli- $Q$ 净化系统 (Millipore 公司) 制备的去离 子水。固相萃取柱 C18、PS、PEP $(500 \mathrm{mg} / 6 \mathrm{~mL}$, 天 津博纳艾杰尔科技公司 ), HLB (500 mg/6 mL, 美国 Waters 公司)。

\section{2 标准溶液的配制}

准确称取苯并三唑、5-甲基苯并三唑、5,6-二甲 基苯并三唑、5-氯-苯并三唑各 $20.0 \mathrm{mg}$, 移取苯并噻 唑 $16.5 \mu \mathrm{L}$, 置于 $100 \mathrm{~mL}$ 容量瓶中, 用甲醇定容, 得 $200 \mathrm{mg} / \mathrm{L}$ 混合标准储备液。临用时,用流动相逐级 稀释成质量浓度分别为 $0.064 、 0.32 、 1.60 、 8.00$ 、
$40.00 、 80.00 \mathrm{mg} / \mathrm{L}$ 的系列浓度的混合标准溶液。

\section{3 色谱条件}

ZORBAX SB-C18 色谱柱 $(250 \mathrm{~mm} \times 4.6 \mathrm{~mm}$, $5 \mu \mathrm{m}$; Agilent 公司); 流动相为甲醇-水 $(55: 45, \mathrm{~V} /$ $\mathrm{v})$, 流速 $1 \mathrm{~mL} / \mathrm{min}$; 紫外光检测波长 $254 \mathrm{~nm}$; 进样 量 $10 \mu \mathrm{L}$; 柱温 $20{ }^{\circ} \mathrm{C}$ 。

\section{4 样品的制备}

实际水样 $(500 \mathrm{~mL})$ 上样前先过 $0.45 \mu \mathrm{m}$ 滤 膜,用 $0.1 \mathrm{~mol} / \mathrm{L}$ 盐酸调节 $\mathrm{pH}$ 至 $5 \sim 6$, 然后用 $\mathrm{HLB}$ 固相萃取柱 $(500 \mathrm{mg} / 6 \mathrm{~mL})$ 进行富集净化。上样前 固相萃取柱依次用甲醇 $(5 \mathrm{~mL} \times 3)$ 、水 $(5 \mathrm{~mL} \times 3)$ 活化。样品溶液以 $5 \mathrm{~mL} / \mathrm{min}$ 的流速上样, 然后用 4 $\mathrm{mL} 30 \%$ 甲醇水溶液淋洗, 最后用 $4 \mathrm{~mL}$ 甲醇洗脱。 洗脱液经温和氮气流吹至约 $0.10 \mathrm{~mL}$, 然后用 $55 \%$ 甲醇水溶液定容至 $1 \mathrm{~mL}$, 供 HPLC 测定。

\section{2 结果与讨论}

\subsection{HPLC 条件的确定}

为了获得良好的分离度, 本文考察了 Agilent 公 司的 ZORBAX SB-C18 $(250 \mathrm{~mm} \times 4.6 \mathrm{~mm}, 5$ $\mu \mathrm{m}) 、$ ZORBAX Eclipse XDB-C18 ( $150 \mathrm{~mm} \times 4.6$ $\mathrm{mm}, 5 \mu \mathrm{m})$ 两种色谱柱和不同比例的乙腈-水、甲 醇-水流动相对目标化合物分离度的影响。最终确 定采用 $250 \mathrm{~mm}$ 长的 ZORBAX SB-C18 色谱柱, 流 动相为 $55 \%$ 的甲醇水溶液,等度洗脱。在上述条件 下 5 个目标化合物得到基线分离(见图 1)。

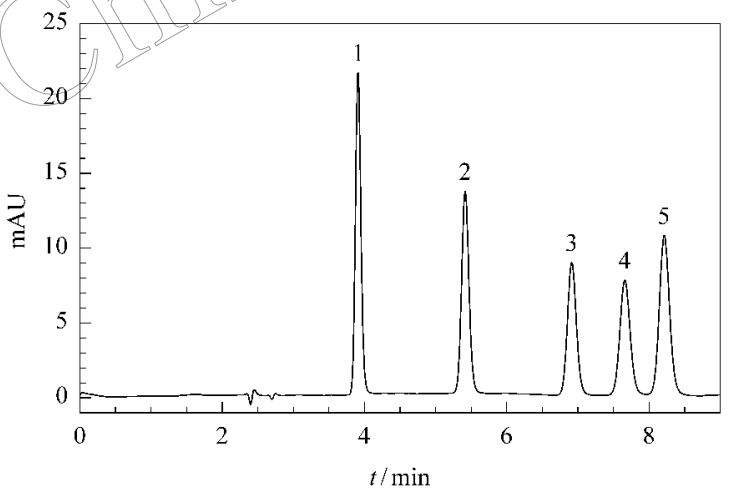

图 1 混合标准溶液的色谱图

Fig. 1 Chromatogram of a mixed standard solution

Chromatographic conditions: mobile phase, methanol-water (55:45, v/v); flow rate, $1 \mathrm{~mL} / \mathrm{min}$; detection wavelength, $254 \mathrm{~nm}$; column temperature, $20{ }^{\circ} \mathrm{C}$.

Peak identifications: 1 . 1H-benzotriazole (BT) ; 2. 5-methyl$1 H$-benzotriazole ( 5 -M-BT) ; 3. 5-chloro- $H$-benzotriazole ( 5 Cl-BT) ; 4. 5, 6-dimethyl-1 $H$-benzotriazole ( 5, 6-DM-BT) ; 5 . benzothiazole (BTH).

\section{2 固相萃取条件的优化}

\subsection{1 萃取柱的选择}

考察了 4 种商品化的固相萃取柱 C-18、HLB、 
PS 及 PEP 对目标化合物的萃取效率, 结果见图 2。 从图 2 中可以看出 C-18 柱仅对 BTH 的回收率大于 $80 \%$,对其余 4 种目标化合物的回收率均较低。PS 和 PEP 对 4 种苯并三唑类目标化合物的回收率均 较高,但对苯并噻唑的回收率不到 10\%。只有 HLB 柱对 5 种目标化合物的回收率均较高。因此后续实 验均采用 HLB 固相萃取柱处理样品。

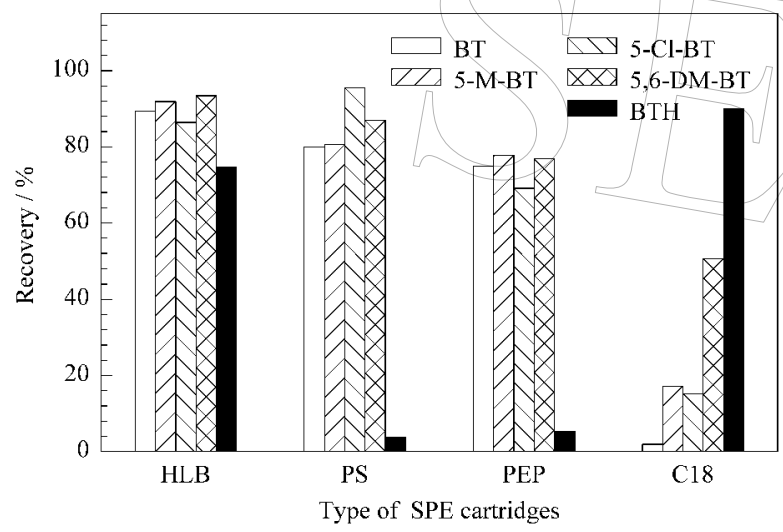

图 2 萃取柱类型对目标化合物回收率的影响

Fig. 2 Recoveries of the analytes obtained by different SPE cartridges

SPE conditions: washing with $5 \mathrm{~mL}$ water; elution with $5 \mathrm{~mL}$ methanol.

\subsection{2 上样体积对回收率的影响}

以 HLB 为固相萃取柱, 考察了上样体积分别为 $100 、 200 、 300 、 500$ 及 $700 \mathrm{~mL}$ 含目标化合物 $10 \mu \mathrm{g} / \mathrm{L}$ 的水溶液时对目标化合物回收率的影响。结果表 明,在考察的体积范围内目标化合物的回收率变化 很小,没有发生穿透现象, 因此实际样品测定时可以 采用大体积上样进行富集。

\subsection{3 淋洗溶剂和洗脱溶剂种类对回收率的影响}

固相萃取的目的是在富集浓缩目标化合物的同 时,也尽量除去其他干扰化合物, 以提高分析的准确 度。为了消除其他化合物的干扰作用,考察了 $4 \mathrm{~mL}$ 不同甲醇含量的水溶液作淋洗溶剂对目标化合物的 淋洗效果。从图 3 中看出, 5,6-DM-BT、5-Cl-BT 和 BTH 的保留较强,即使用 $60 \%$ 甲醇水溶液淋洗仍没 有被洗脱下来。而 BT 在淋洗溶剂的甲醇含量大于 $30 \%$ 时即开始被洗脱下来。因此实验中选择用 4 $\mathrm{mL} 30 \%$ 甲醇水溶液为淋洗溶剂。

为了获得理想的回收率, 考察了分别以 $0.5 、 1$ 、 $2 、 3 、 4$ 及 $5 \mathrm{~mL}$ 甲醇为洗脱溶剂对目标化合物回收 率的影响。结果 (见图 4) 表明, 当甲醇用量小于 2 $\mathrm{mL}$ 时, 目标化合物不能被完全洗脱; 当甲醇用量大 于 $4 \mathrm{~mL}$ 时, 目标化合物的回收率基本恒定。因此 实验中确定洗脱溶剂甲醇的用量为 $4 \mathrm{~mL}^{\circ}$

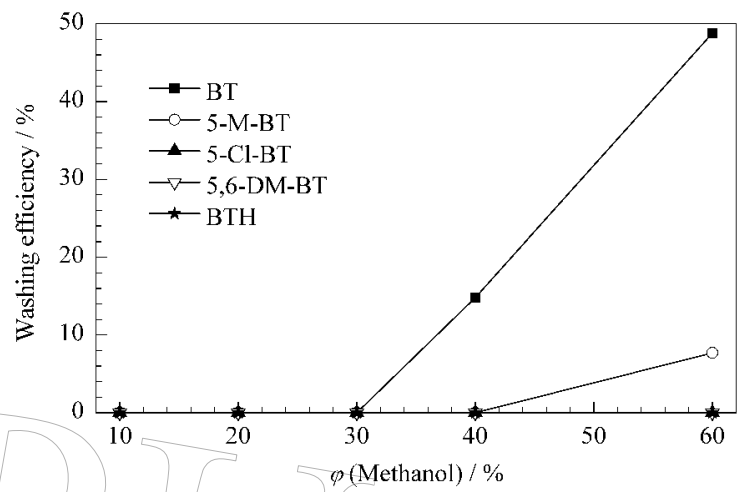

图 3 淋洗溶剂 (甲醇水溶液) 中甲醇含量对淋洗效果的影响

Fig. 3 Effect of methanol content in methanolwater washing solution on the washing efficiency

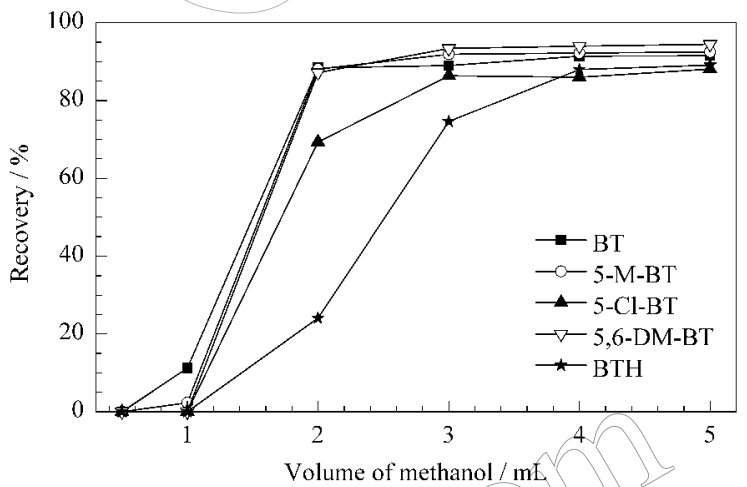

图 4 洗脱溶剂甲醇的体积对回收率的影响

Fig. 4 Effect of volume of methanol as eluent on the recovery

\subsection{4 上样溶液 $\mathrm{pH}$ 值对回收率的影响}

图 5 是不同 $\mathrm{pH}$ 值的上样溶液对目标化合物回 收率的影响。结果表明, 上样溶液 $\mathrm{pH}$ 值的变化对 苯并噻唑的回收率影响不大。 $\mathrm{pH}>7.5$ 时, BT、5M-BT 、 5,6-DM-BT 及 5-Cl-BT 的回收率开始下降。 这可能是因为在碱性条件下苯并三唑及其衍生物生 成了盐, 导致固相萃取柱对它们的富集能力下降。 因此, 为确保回收率不受损失, 在固相萃取之前先用 盐酸调节样品的 $\mathrm{pH}$ 为 $5 \sim 6$ 之后再上样。

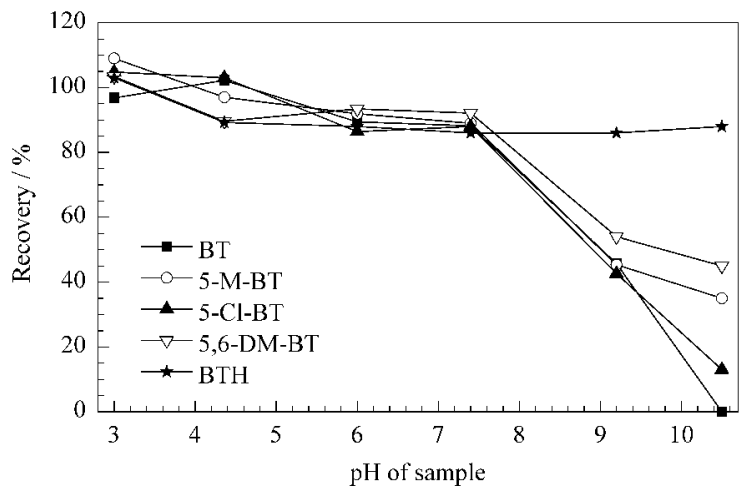

图 5 上样溶液 $\mathrm{pH}$ 值对回收率的影响

Fig. 5 Effect of $\mathrm{pH}$ of the sample on the recovery 


\section{3 方法的线性范围、回收率、精密度及检出限}

在优化的色谱条件下,考察了 5 种目标化合物在 $0.064 \sim 80 \mathrm{mg} / \mathrm{L}$ 范围内的线性关系, 结果表明在上 述范围内目标化合物的线性关系良好 (相关系数 $\geqslant$ 0.9999 , 见表 1$)$ 。以信噪比 $(S / N)$ 为 3 时 5 种目标
化合物的质量浓度为仪器检出限, 得到的结果为 1.9 $\sim 3.2 \mu \mathrm{g} / \mathrm{L}_{\text {。 }}$ 在空白自来水样中添加高、中、低 3 个 浓度水平的混合标准溶液, 每个浓度水平的样品平行 分析 3 次, 5 种目标化合物的加标回收率为 $87.8 \%$ $125.6 \%$, 相对标准偏差 (RSD) 为 $0.4 \% \sim 9.4 \%$ 。

表 1 BT、5-M-BT、5,6-DM-BT、5-Cl-BT、BTH 的线性范围、线性方程、相关系数、加标回收率及仪器检出限 ( $n=3$ )

Table 1 Linear ranges, regression equations, correlation coefficients $(r)$, spiked recoveries and instrumental limits of detection (LOD, $S / N=3$ ) of BT, 5-M-BT, 5,6-DM-BT, 5-Cl-BT and BTH $(n=3)$

\begin{tabular}{|c|c|c|c|c|c|c|c|}
\hline Analyte & Linear range/ $(\mathrm{mg} / \mathrm{L})$ & Linear equation & $r$ & Spiked/ $(\mu \mathrm{g} / \mathrm{L})$ & Recovery/\% & $\mathrm{RSD} / \%$ & $\mathrm{LOD} /(\mu \mathrm{g} / \mathrm{L})$ \\
\hline \multirow[t]{2}{*}{ BT } & $0.064-80$ & $A=29.735 C+0.134$ & 1.0000 & 0.2 & 96.3 & 6.0 & 1.9 \\
\hline & & & & $\begin{array}{c}4 \\
16\end{array}$ & $\begin{array}{l}96.1 \\
97.8\end{array}$ & $\begin{array}{l}5.3 \\
0.6\end{array}$ & \\
\hline \multirow[t]{2}{*}{ 5-M-BT } & $0.064-80$ & $A=24.612 C-0.076$ & 0.9999 & 0.2 & 93.2 & 6.5 & 3.2 \\
\hline & & & & $\begin{array}{c}4 \\
16\end{array}$ & $\begin{array}{l}95.9 \\
95.8\end{array}$ & $\begin{array}{l}5.7 \\
0.7\end{array}$ & \\
\hline \multirow[t]{3}{*}{ 5-Cl-BT } & $0.064-80$ & $A=19.728 C+0.319$ & 0.9999 & 0.2 & 125.6 & 2.8 & 1.9 \\
\hline & & & & 4 & 93.4 & 5.1 & \\
\hline & & & & 16 & 95.9 & 0.4 & \\
\hline \multirow[t]{3}{*}{$5,6-\mathrm{DM}-\mathrm{BT}$} & $0.064-80$ & $A=19.602 C+0.892$ & 0.9999 & 0.2 & 87.8 & 5.5 & 3.2 \\
\hline & & & & 4 & 93.9 & 5.9 & \\
\hline & & & & 16 & 94.5 & 3.3 & \\
\hline \multirow[t]{3}{*}{ BTH } & $0.064-80$ & $A=27.156 C+0.789$ & 1.0000 & 0.2 & 124.4 & 9.4 & 2.2 \\
\hline & & & & 4 & 94.3 & 6.5 & \\
\hline & & & & 16 & 95.7 & 0.8 & \\
\hline
\end{tabular}

$A$ : peak area; $C$ : mass concentration, $\mathrm{mg} / \mathrm{L}$.

\section{4 实际水样的测定}

用建立的方法对大连自来水及辽河人海口的水 样进行了测定。辽河人海口水样采自辽河人海口表 层水,采样日期为 2012 年 5 月 30 日,于 $4{ }^{\circ} \mathrm{C}$ 储存。 自来水为 2012 年 8 月 27 日采自实验室。分析结果 表明,5 种目标化合物在自来水中均未检出; 在辽河 人海口的水样中检出苯并三唑 $(1.072 \mu \mathrm{g} / \mathrm{L}) 、 5$-甲 基苯并三唑 $(0.691 \mu \mathrm{g} / \mathrm{L}) 、 5$-氯-苯并三唑 $(0.837$ $\mu \mathrm{g} / \mathrm{L})$ 及苯并噻唑 $(5.695 \mu \mathrm{g} / \mathrm{L})$ 。辽河流域分布 在沈阳、抚顺、鞍山、本溪及营口等大中城市,这些城 市中有许多大中型企业, 且人口密度大, 大量的工业 废水及生活污水排放到辽河中, 这是导致辽河水中 苯并三唑及苯并噻唑污染的主要原因。

\section{3 结论}

本文采用固相萃取-高效液相色谱建立了地表 水中苯并三唑、5-甲基苯并三唑、5,6-二甲基苯并三 唑、5-氯-苯并三唑及苯并噻唑的分析方法。该方法 简便快速,线性关系、回收率及重现性等方法学指标 均较好,可用于我国地表水中苯并噻唑、苯并三唑及 其衍生物的环境监测分析。

\section{参考文献：}

[1] Patsalides E, Robards K. J Chromatogr, 1985, 331: 149

[2] Cancilla D A, Baird J C, Geis S W, et al. Environ Toxicol Chem, 2003, 22: 134
[3] Gruden C L, Dow S M, Hernandez M T. Water Environ Res, 2001, 73: 72

[4] Gradwell M H S, McGill W J. J Appl Polym Sci, 1995, 58 : 2185

[5] Ajmal M, Mideen A S, Quraishi M A. Corrosion Sci, 1994, 36: 79

[6] Achim K, Martin J, Thorsten R. J Chromatogr A, 2004, 1058: 81

[7] Stefan W, Thorsten R. Anal Chem, 2005, 77: 7415

[8] Achim K, Martin J, Thorsten R. Environ Sci Technol, 2005, 39: 3792

[9] Cancillia D A, Baird J C, Rosa R. Bull Environ Contam Toxicol, 2003, $70: 868$

[10 ] Janna H, Scrimshaw M D, Williams R J, et al. Environ Sci Technol, 2011, 45: 3858

[11] Susan D R. Anal Chem, 2008, 80: 4391

[12] Susan D R. Anal Chem, 2010, 82: 4765

[13] Susan D R. Anal Chem, 2012, 84: 769

[14] Natural Resource Management Ministerial Council, Environment Protection and Heritage Council, and National Health and Medical Research Council ( NRMMC-EPHCNHMRC). Australian Guidelines for Water Recycling. Augmentation of Drinking Water Supplies. Adelaide: NRMMCEPHC-NHMRC, 2008

[15] Liu Y S, Ying G G, Shareef A, et al. J Chromatogr A, $2011,1218: 5328$

[16] Carmen D, Carolina R C, Josep M B. J Chromatogr A, $2012,1230: 117$

[17] Zhang Z F, Ren N Q, Li Y F. Environ Sci Technol, 2011, 45: 3909

[18 ] Teresa Pena M, Vecino-Bello X, Carmen Casais M, et al. Anal Bioanal Chem, 2012, 402: 1679

[19] Carpinteiro I, Abuin B, Ramil M, et al. Anal Bioanal Chem, 2012, 402: 2471

[20 ] Leerdam J A, Hogenboom A C, Kooi M M E. Int J Mass Spectrom, 2009, 282 : 99 